\title{
Analysis of the attenuative behaviour of accelerated cement based materials through a series of
} ultrasound Pulse Echo measurements

\author{
Nabil Khatib ${ }^{a}$, El Houssaine Ouacha ${ }^{a}$, Bouazza Faiz ${ }^{a}$, Mohamed Ezzaidia and Hicham Banouni ${ }^{a}$
}

${ }^{a}$ Laboratory of Metrology and Information Processing, Ibn Zohr University, Faculty of science, B.P. 8106, Agadir, Morocco

\begin{tabular}{l}
\hline A R T I C L EI N F O \\
\hline Article history: \\
Received 26 December, 2018 \\
Accepted 26 February 2019 \\
Available online \\
11 April 2019 \\
\hline Keywords: \\
Non-destructive testing \\
Ultrasound \\
Pulse echo method \\
Mortar \\
Alkaline Accelerator
\end{tabular}

\section{Introduction}

Currently, the non-destructive techniques are the most used methods for quality control in civil engineering. Among these techniques, the ultrasound measurements technique has been greatly developed during this last two decades, in order to monitor the hydration process and to assess the structural performance of concrete, shotcrete and cement-mortars (Bita et al. 2017; Reinhardt \& Grosse 2004; Lotfi et al. 2013). Thus, non-destructive ultrasound measurements clearly offer advantages in this field, since they can be performed on both mortar and concrete (shotcrete), as opposed to the traditional laboratory tests on cement paste such as Gillmore needles (ASTM C1102-88, 2000) or a Vicat needle (Melbye. 1996). Moreover, the comprehension of the different stages of cement hydration, mainly the setting and hardening process is also of great importance for the study of the effect of admixtures and cement replacing additives on the development of the physical, mechanical and durability properties of hardened cementitious materials. A lot of research has already been done in order to evaluate the sensitivity of the velocity of US longitudinal waves (P-wave velocity) to the presence of some admixtures. Robeyst et al. (2008) focused on the application of the transmission method with compression waves (P-waves) to monitor the setting of concrete containing blast-furnace slag by

\footnotetext{
* Corresponding author.
}

E-mail addresses: nabil.khatib@edu.uiz.ac.ma (N. Khatib) 
following the development of P-wave velocity in time (concrete age). De Belie et al. (2005) found that the change of ultrasound P-wave velocity in time is sensitive to setting behaviour of the alkaline and alkali-free accelerating admixtures. Also, Zhu et al. (2011) showed that the P-wave velocity is strongly affected by air voids in early ages cement pastes, and indicated that one percent of air voids in fresh cement paste reduce the P-wave velocity from $1500 \mathrm{~m} / \mathrm{s}$ to about $200 \mathrm{~m} / \mathrm{s}$. The measurements of P-wave velocity have been made more practical due to the ultrasound FreshCon device developed by Reinhardt and Grosse at the Institute of Construction Materials of the university of Stuttgart, which was described in many publications (Reinhardt \& Grosse 2004; Reinhardt et al. 2000, 2001).

In recent years, new US-parameters have been used to characterize and evaluate the effect of accelerating admixtures on setting and hardening of concrete (shotcrete) and cement mortars, which consider the entire received signals. Measurement of such parameters is done using many advanced methods, mostly the ultrasound wave transmission and pulse echo (wave reflection) method. Applying the wave transmission method and P-waves, De Belie et al. (2005) indicated that US-energy can be used to study the influence of different accelerating admixtures and cement types on setting and hardening behaviour of shotcrete. Grosse and Reinhardt (2000) affirmed that more details on the hydration process can be obtained by using Fast Fourrier Transform (FFT). Numerous attempts have been made during this last years on using US-parameters involving the frequency domain of obtained signals, to monitor setting and hardening of cementitious materials. Trtnik et al. (2008) used a new parameter, labeled as TG parameter, which is defined as a dimensionless ratio between maximum amplitude of two dominant frequency ranges that appears in frequency spectrum of received ultrasound signals, to monitor the setting process of cement pastes, and find that the combined use of TG parameter and the P-wave velocity represents an comprehensive ultrasound method to monitor the formation of structure of cement based materials at early ages. Also, Lotfi et al. (2009) and Khatib et al. (2018) presented the possibility of using the frequency attenuation coefficient to study the influence of sand size on hardening behaviour of mortar pastes.

In this study, the effects of alkaline accelerator for shotcrete and its dosage on early age cement pastes were evaluated by following the evolution of the ultrasound attenuation coefficient during the setting and the hardening period. Therefore, a comprehensive experimental work was performed in which the ultrasound pulse echo method was used and P-waves were generated and received by an immersion transducer of $1 \mathrm{MHz}$ central frequency. Also, the accelerated cement based materials were examined by following the evolution of the different echoes on the ultrasound histograms presented in time domain. In addition, to measure the degree of porosity as well as its effect on the resistivity of our evaluated mortar samples, the ultrasound density parameter and young modulus were represented in time domain.

\section{Materials and methods}

\subsection{Hardware}

The schematic presentation of ultrasound experimental system used to monitor mortar samples in immersing testing is represented in Fig. 1.

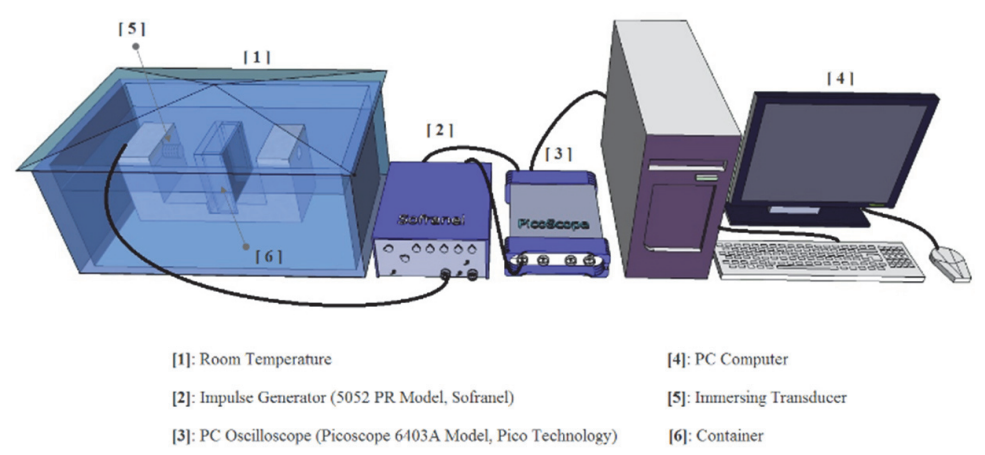

Fig. 1. Schematic presentation of the ultrasound test setup 


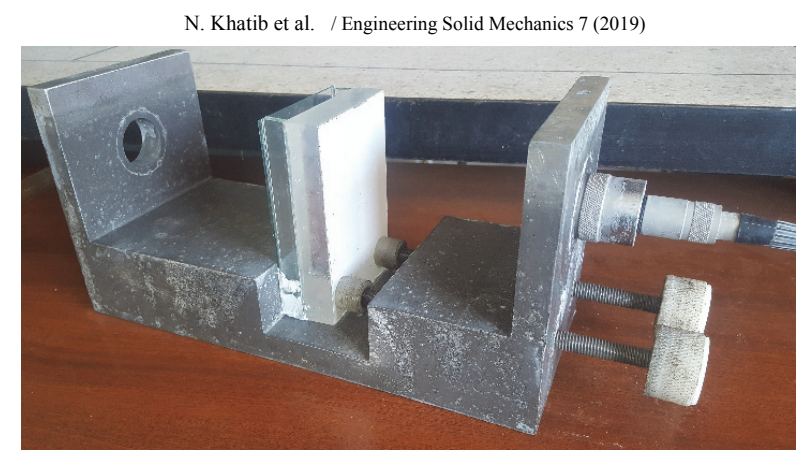

Fig. 2. Close view of the container mounted in the steel carrier

The US measurements on mortar pastes during setting and hardening period were performed with a commercially available ultrasound instrument, which consists of a broadband p-wave immersing transducer used successively as transmitter and as a receiver of ultrasound and an impulse generator (5052 PR Model, Sofranel, France). This immersion transducer is a panametric's (V 302 Model) transducer with a central frequency $1 \mathrm{MHz}$. For further processing and quantitative evaluation using a developed software, the received ultrasound signals are captured by an analog-to-digital conversion process, which is taken place into a PC Oscilloscope (Picoscope 6403A Model, Pico technology) that is a transient recorder with $12 \mathrm{Bit}$ amplitude resolution. The digitizer operates in a real time sampling mode, that captures an ultrasound waveform signal during on repetition time, for a simple pulse shape being sampled at a frequency of $625 \mathrm{MHz}$, when $\Delta t=1.6 \cdot 10^{-9} \mathrm{~s}$ is the time spacing between samples. Also, the PC Oscilloscope averages the received ultrasound signals on twenty repetition cycles to reduce electronic noise. The instrument was connected to a PC and all the data were taken and stored automatically every $5 \mathrm{~min}$. As shown in Fig. 1 and 2, the transducer and the container filled with mortar sample, are both mounted in the steel carrier before being fully submerged in a room temperature. The steel carrier and the container are dimensioned in the laboratory before being manufactured by a subcontractor. Fig. 3 and 4 show the results of the development of the steel carrier and the container respectively.

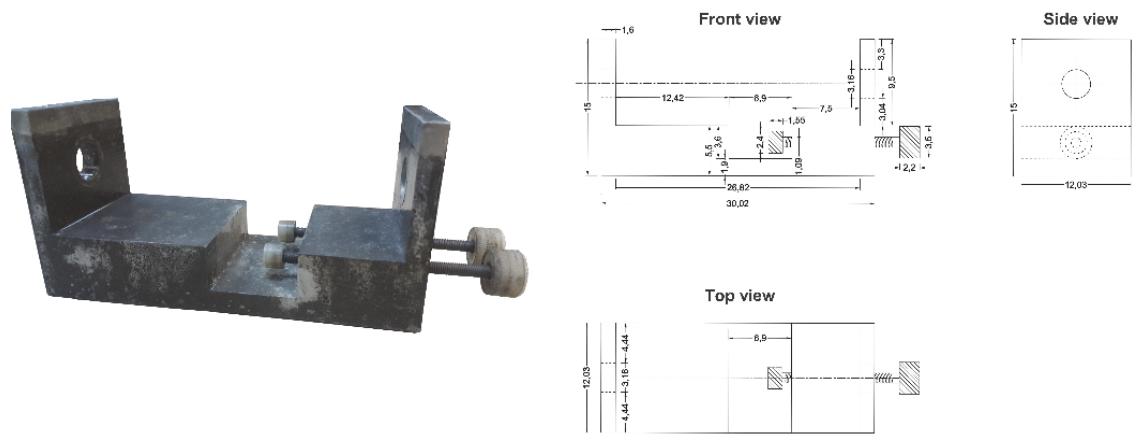

Fig. 3. Views of the steel carrier

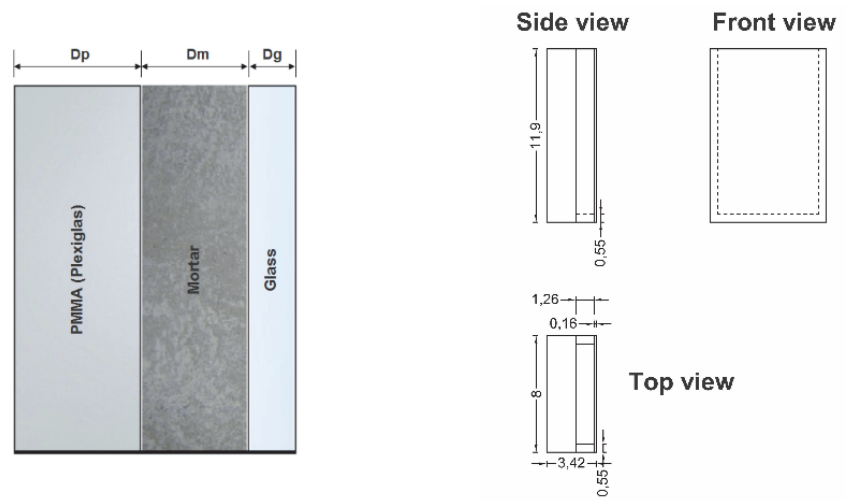

Fig. 4. Views of the container 


\subsection{Measurement procedure}

The ultrasound Pulse echo measurement method is used to evaluate mortar samples, filling a parralipipeted container, in immersion testing. The container is excited by a broadband P-wave transducer used successively as a transmitter and a receiver of ultrasounds. This transducer is characterized by a central frequency of $1 \mathrm{MHz}$. The immersion transducer and the container, are fixed in a steel carrier before being fully submerged in a room temperature filled with water and regulated at ambient temperature of $25^{\circ} \mathrm{C}$. The container consists of one polymethacrylate (PMMA, known under the Plexiglas commercial name) wall and a glass wall as is shown in Fig. 5.

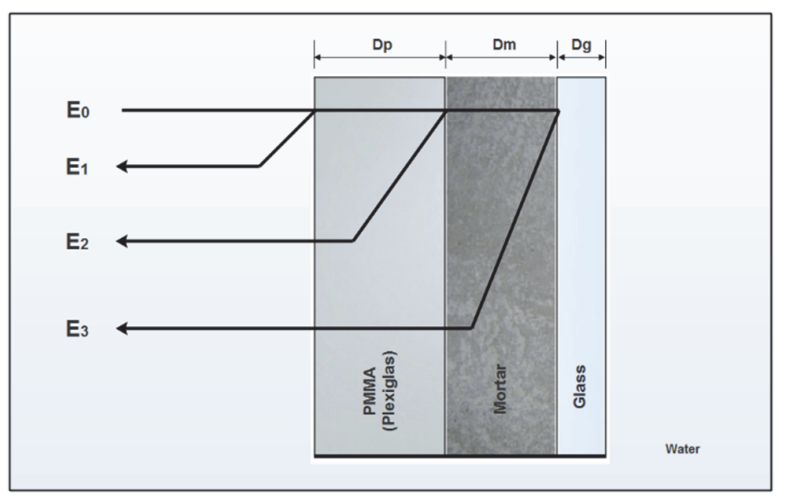

Fig. 5. Container's media and Ultrasound Signal's paths

The aim of this present work is to follow the evolution of frequency dependent attenuation coefficient, $\alpha(f)$, as a function of mortar age. This coefficient is measured using backscattered signals from the different boundaries between the container's media. As showed in Figs. 5 and 6, the principal echoes generated by the boundaries of the container's media, those needed to evaluate the attenuation caused by the mortar sample, are as follow

- $\mathrm{A}_{2}$ corresponds to the spectral amplitude of echo E2 of the backscattered signal at the boundary between the PMMA plate and the mortar sample.

- $\mathrm{A}_{3}$ corresponds to the spectral amplitude of echo E3 of the backscattered signal at the boundary between the mortar sample and the glass plate.

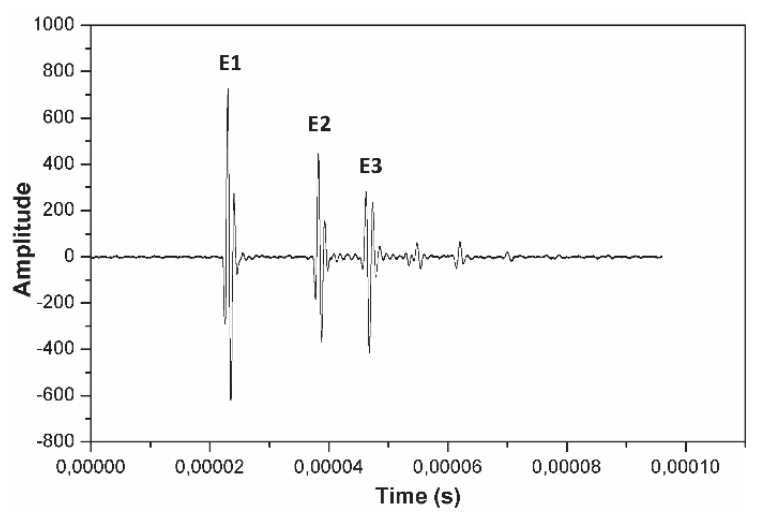

Fig. 6. Principal pulse echoes reflected at boundaries between the different container's media

\subsection{Software}

The software programs developed at the Laboratory of Metrology and Information Processing at the University Ibn Zohr of Agadir, using LabView software, visualize every recorded backscattered signal and its frequency spectrum as shown is Fig. 7. The whole experiment takes 72 hours and as a recording interval, 5 min was chosen. An offline version of the software program allows further analysis and 
processing of signals data after the test. Thus, the offline version allows the measurement of ultrasound parameters after the isolation of the different echoes using isolation algorithms.

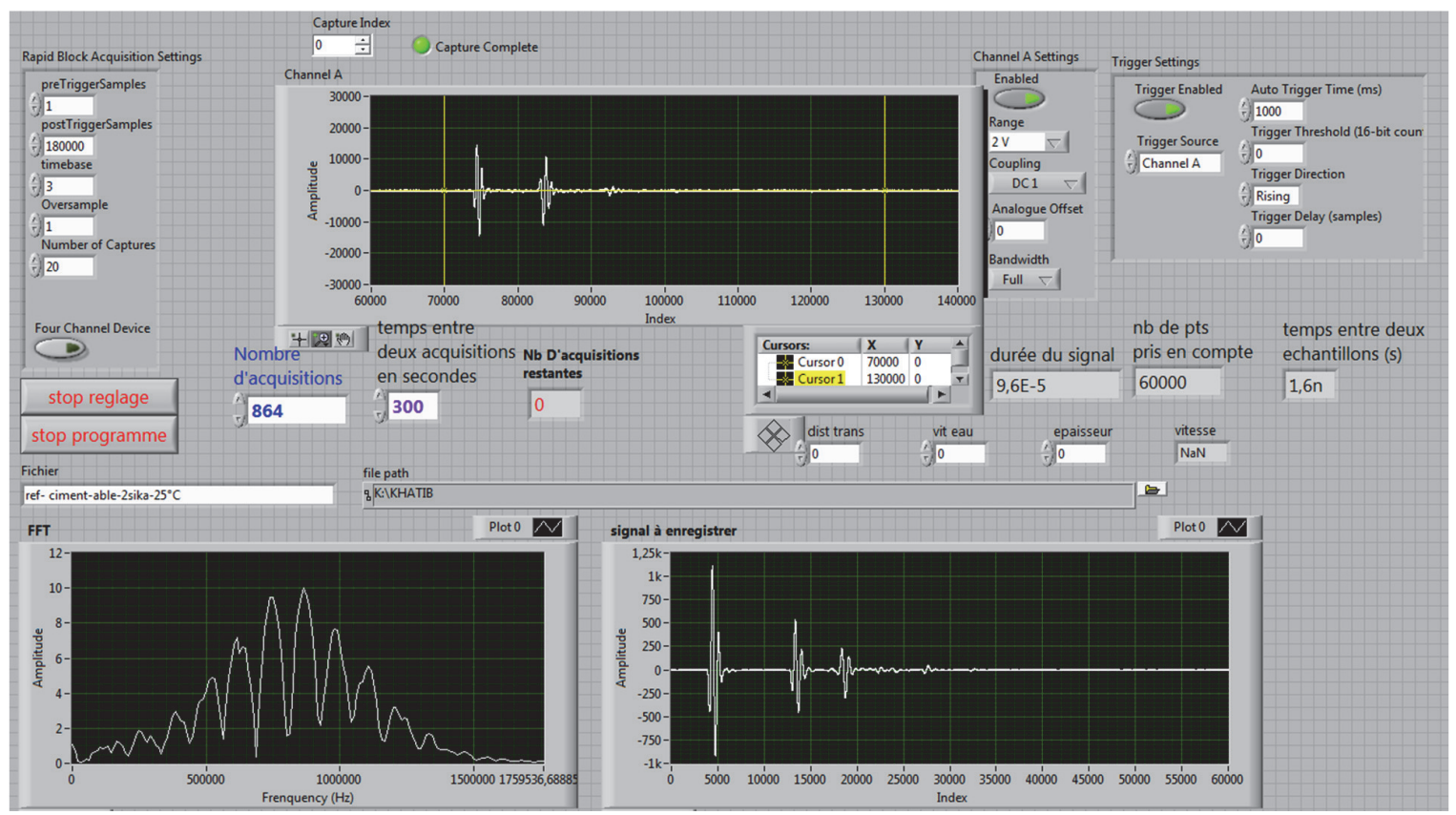

\subsection{Attenuation coefficient}

Fig. 7. Screen-shot of the LabView Software

The immersion transducer used, generates P-waves. The generated ultrasound longitudinal P-waves travel in the investigated material in one spatial dimension, $\mathrm{x}$. form

The one-dimensional plane wave equation can be shown to have a solution, in $+x$ direction, of the

$$
f\left(t-\frac{x}{v}\right)=\frac{1}{2 \pi} \int_{-\infty}^{+\infty} F(\omega) \cdot \exp \left(i \omega\left(\frac{x}{v}-t\right)\right) d \omega
$$

The Fourier transform of the solution $f\left(t-\frac{x}{v}\right)$, is of the form

$$
\int_{-\infty}^{+\infty} f\left(t-\frac{x}{v}\right) \cdot \exp (i \omega t) d t=F(\omega) \cdot \exp \left(i \omega \frac{x}{v}\right)
$$

where

$$
F(\omega)=\int_{-\infty}^{+\infty} f(t) \cdot \exp (i \omega t) d \omega
$$

is the Fourier transform of $f(t)$.

Eq. 1 shows that the solution of the $\mathrm{P}$-wave equation propagating in $+\mathrm{x}$ direction is the superposition, over all frequencies, of a harmonic plane wave of the form

$$
p=A(\omega) \cdot \exp \left(i \omega\left(\frac{x}{v}-t\right)\right)
$$

where $\omega$ is related to the frequency, $f$, in Hertz, through $\omega=2 \pi f, v$ the material wave speed (velocity) and $x$ the distance traveled. The amplitude $A$, of the plane wave, is just proportional to the pressure wave, i.e., $A(\omega)=F(\omega) / 2 \pi$. Thereby, we will only consider the response to a harmonic wave, since the general solution, over all frequencies, is obtained by performing the inverse Fourier transform. 
The above solution of the equation of wave motion have been for ideal and lossless media. At ultrasonic frequencies, however, there are material dependent losses that cause waves to attenuate as they propagate. The attenuation losses are characterized in a simple, ad hoc fashion (Schmerr 2016). According to Schmerr (2016), the effects of attenuation are modeled by an exponential factor that contains a frequency dependent attenuation coefficient, $\alpha(f)$.

Consider, the P-wave traveling through an attenuating material. The amplitude of this wave will change as it propagates. If we let $A_{0}$ be the amplitude of this wave at $x=x_{0}$ and $A_{1}$ the amplitude at $x=x_{1}$ where $x_{1}>x_{0}$ then in general $A_{1}<A_{0}$ due to losses in the propagating medium. These losses are expressed in the form

$$
\frac{A_{1}}{A_{0}}=\exp (-\alpha(f) \cdot d)
$$

where $d=x_{1}-x_{0}$ is the distance traveled in the material. This attenuation coefficient is measured in Nepers/unit length $(\mathrm{Np} / \mathrm{m})$, when Neper is a dimensionless quantity.

At a given frequency $f$, i.e. $\omega=2 \pi f$, the solution of wave propagating equation, is of the form

$$
A(\omega) \cdot \exp (-\alpha(f) \cdot x) \cdot \exp \left(i \omega\left(\frac{x}{v}-t\right)\right)
$$

In our case, the immersing transducer generates ultrasound waves which propagate in the container filled with the evaluated mortar sample. The generated ultrasound signal has an amplitude $A^{*}(f)$ at a frequency $f$. Before reaching the front of the PMMA plate, ultrasound wave propagates in water medium. The attenuation of water is to be considered. It has been measured as function of temperature Pinkerton (1947). At room temperature regulated at $25^{\circ} \mathrm{C}$, the attenuation coefficient of water has been measured as Pinkerton (1947):

$$
\alpha_{w}(f)=27.9365 \cdot 10^{-5} \cdot f^{2} \mathrm{~Np} / \mathrm{m}
$$

The monochromatic solution of wave propagation equation is expressed as follow:

$$
A^{*}(f) \cdot \exp \left(-\alpha_{w}(f) \cdot d_{w}\right) \cdot \exp \left(i w\left(\frac{x}{V_{w}}-t\right)\right)
$$

where $V_{w}$ is the ultrasound velocity in water medium and $d_{w}$ the distance between the front of the transmitting transducer and the front of the PMMA.

After the first reflection at the front of the PMMA plate, the reflected wave (propagating in the $-\mathrm{x}$ direction) have a monochromatic solution in the form:

$$
A^{*}(f) \cdot R_{w / p g} \cdot \exp \left(-2 \alpha_{w}(f) \cdot d_{w}\right) \cdot \exp \left(i w\left(\frac{x}{V_{w}}+t\right)\right)
$$

and

$$
R_{w / p g}=\frac{Z_{p g}-Z_{w}}{Z_{p g}+Z_{w}}
$$

where $Z_{p g}$ and $Z_{w}$ are respectively the acoustic impedances of the PMMA (Plexiglas) and water media.

The Fourier transform of the wave propagation solution is:

$$
A^{*}(f) \cdot R_{w / p g} \cdot \exp \left(-2 \alpha_{w}(f) \cdot d_{w}\right) \cdot \exp \left(i w \frac{x}{V_{w}}\right)
$$

With 
$A_{1}=A^{*}(f) \cdot R_{w / p g} \cdot \exp \left(-2 \alpha_{w}(f) \cdot d_{w}\right)$

The transmitted wave into the PMMA medium has a monochromatic solution as follow:

$$
A^{*}(f) \cdot T_{w / p g} \cdot \exp \left(-\alpha_{w}(f) \cdot d_{w}\right) \cdot \exp \left(-\alpha_{p g}(f) \cdot x\right) \cdot \exp \left(i w\left(\frac{x}{V_{p g}}-t\right)\right)
$$

With

$$
T_{w / p g}=\frac{2 Z_{w}}{Z_{p g}+Z_{w}}
$$

and $V_{p g}$ the ultrasound velocity in the PMMA medium.

At the boundary between the PMMA and mortar mediums, the amplitude of the Fourier transform of the transmitted wave propagation solution is:

$$
B_{1}=A^{*}(f) \cdot T_{w / p g} \cdot \exp \left(-\alpha_{w}(f) \cdot d_{w}\right) \cdot \exp \left(-\alpha_{p g}(f) \cdot d_{p g}\right)
$$

where $d_{p g}$ is the thickness of the PMMA plate.

By the same process and at each boundary, we found:

$$
A_{2}=A^{*}(f) \cdot T_{w / p g} \cdot R_{p g / m o r} \cdot T_{p g / w} \cdot \exp \left(-2 \alpha_{w}(f) \cdot d_{w}\right) \cdot \exp \left(-2 \alpha_{p g}(f) \cdot d_{P}\right)
$$

and

$$
\begin{gathered}
A_{3}=A^{*}(f) \cdot T_{w / p g} \cdot T_{p g / m o r} \cdot R_{m o r / g l} \cdot T_{m o r / p g} \cdot T_{p g / w} \cdot \exp \left(-2 \alpha_{w}(f) \cdot d_{w}\right) \cdot \exp \left(-2 \alpha_{p g}(f) \cdot d_{p g}\right) \\
\cdot \exp (-2 \alpha(f) \cdot L)
\end{gathered}
$$

where $\alpha(f)$ is the attenuation coefficient in mortar to be evaluated.

So,

$$
\alpha(f)=-\frac{1}{2 L} \cdot \ln \left(\frac{A_{3}}{A_{2}} \cdot \xi_{r e f}\right)
$$

With

$$
\xi_{\text {ref }}=\left|\frac{\left(Z_{\text {mor }}-Z_{p g}\right)\left(Z_{p g}+Z_{\text {mor }}\right)\left(Z_{\text {mor }}+Z_{g l}\right)}{4 Z_{\text {mor }} Z_{p g}\left(Z_{g l}-Z_{\text {mor }}\right)}\right|
$$

\subsection{Preparation of samples}

To allow accurate measurements, starting rapidly after the mix preparation, the experiments were performed on mortar. The reference mortar sample was designed by mixing standard sand extracted from the river of OUED SOUSS, cement powder and water together, with a respect to a particular mix proportions, i.e., the ratios by mass of water-to-cement $(w / c=0.65)$ and of cement-to-sand $(c / s=0.5)$. The cement type tested was Portland composite cement CPJ 45, produced in the cement plant of AIT BAHA, in the region of Agadir, according to the standard Moroccan NM 10.1.004, 2004 (24p). The tested admixture is an alkaline setting accelerator SIKA CIMACCEL NCL2. The accelerator dosage amounted to $0 \%, 1.7 \%, 3.4 \%$ and $5.1 \%$ of the cement weight used in tested mortar.

\section{Results and Discussions}

The attenuative behaviour of accelerated mortar pastes was evaluated. The tested accelerating admixture's dosages amounted to $0 \%, 1.7 \%, 3.4 \%$ and $5.1 \%$ of the cement weight used in tested mortar. The results of the attenuation coefficient measurements for the different mortar pastes are shown in Fig. 8. 


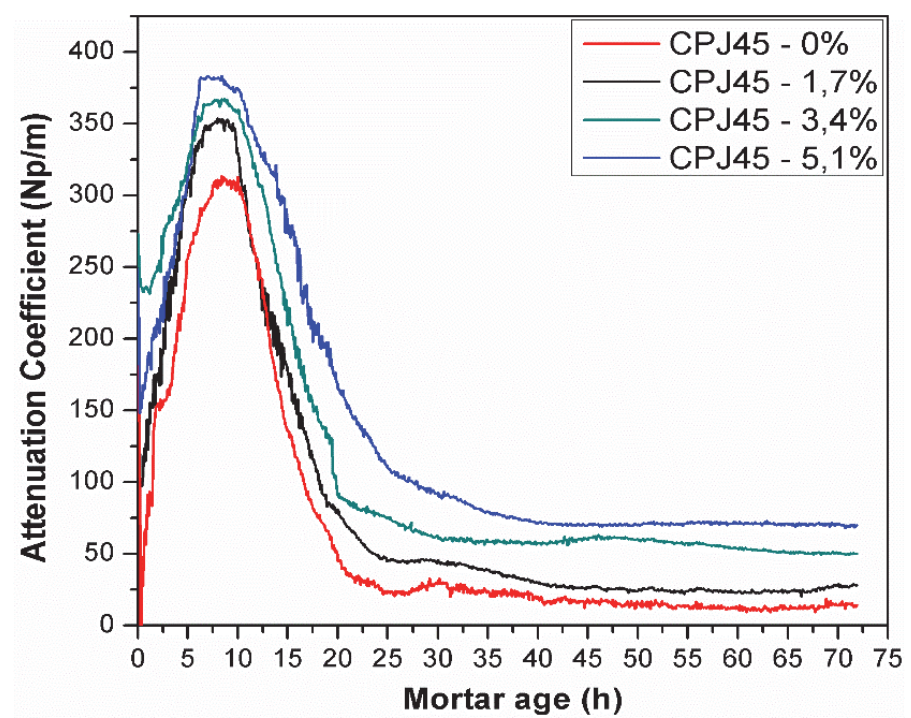

Fig. 8. The evolution of the frequency dependent attenuation coefficient vs. mortar age. Mortar were prepared using the cement type CPJ45 and an alkaline accelerator dosage of $0 \%, 1.7 \%, 3.4 \%$ and $5.1 \%$ of the cement weight used

Before describing the attenuation coefficient evolution, we must take note that in samples with Portland cement, setting is normally completed within $6 \mathrm{~h}$ to $8 \mathrm{~h}$ (De Belie et al. 2005). By following the evolution of attenuation coefficient vs. mortar age, the cement hydration stages have been identified. Thus, four major stages are involved in the hydration of Portland composite cement CPJ 45. The first stage corresponds to the initial dissolution of cement, which results in a rapid release of heat that last for few minutes. The second stage is the dormant period. This stage corresponds to the time of workability, during which the hydration reactions occur slowly. During this stage, the attenuation coefficient began to increase. The third stage is the setting period at which time the cement starts to react rapidly with water. In this period, the attenuation coefficient increases sharply after which a maximum was reached. Reaching this maximum indicates the end of the setting period and the beginning of the hardening phase. Also, the fourth stage is the hardening period, when the rate of hydration reactions slows. During this period, the wave attenuation coefficient decreases sharply after which attenuation coefficient curves levelled off, indicating a steady state.

Thus, the attenuation coefficient measurements are clearly sensitive to the effect of the alkaline accelerator and its dosage on mortar behavior during the setting and the hardening period. An increase in alkaline accelerator dosage causes the attenuation coefficient to reach its maximum at earlier time, compared to mortar samples with less added dosage. Those results validate the role of setting accelerator, which consists in shortening the setting period. However, a stepwise increase in accelerator dosage resulted in increasing values at which the attenuation coefficient curves levelled off, indicating a more attenuative cement-material during the hardening period. This increase in the levelling off value could be due to an expansion of the void volume during the hardening stage of mortar pastes.

More details can be obtained about the stiffening process, the porosity ratio and the resistivity of hardened mortar pastes on the basis of the development of the ultrasound measured density and young modulus in time (mortar age). Thus, using the pulse echo method, the density is measured by the following formula

$$
\rho=\frac{Z}{v}
$$

with $v$ and $Z$ are respectively the P-wave velocity and acoustic impedance in mortar pastes. In the 1D case if the cross-section remains the same $E=Z \cdot v$ with $E$, Young's modulus. 
The changes of ultrasound density and Young's modulus in time (mortar age) for mortar mixes with different dosages of alkaline accelerating admixture are presented respectively in Fig. 9 and Fig. 10.

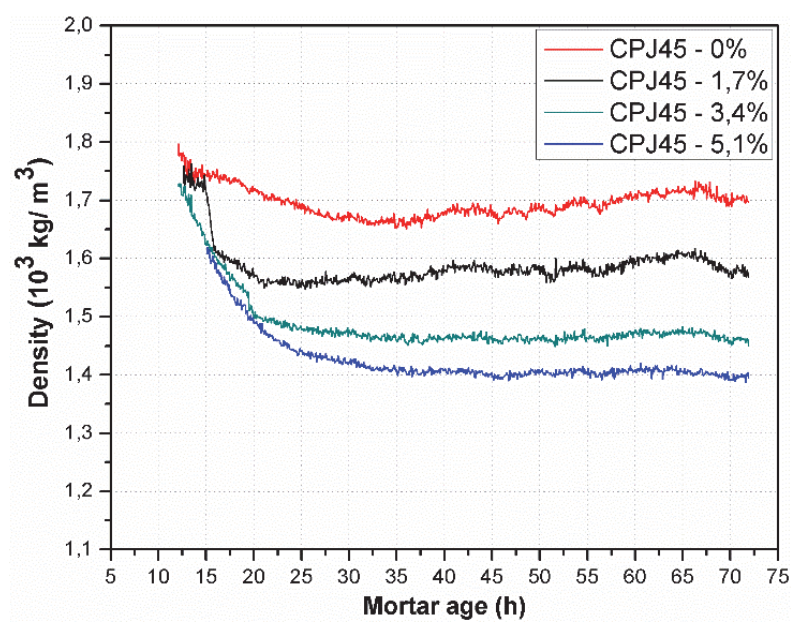

Fig. 9. Ultrasound density vs. mortar age. Mortars were prepared using Portland composite cement CPJ 45 and an alkaline accelerator dosage of $0 \%, 1.7 \%, 3.4 \%$ and $5.1 \%$ of the cement weight used

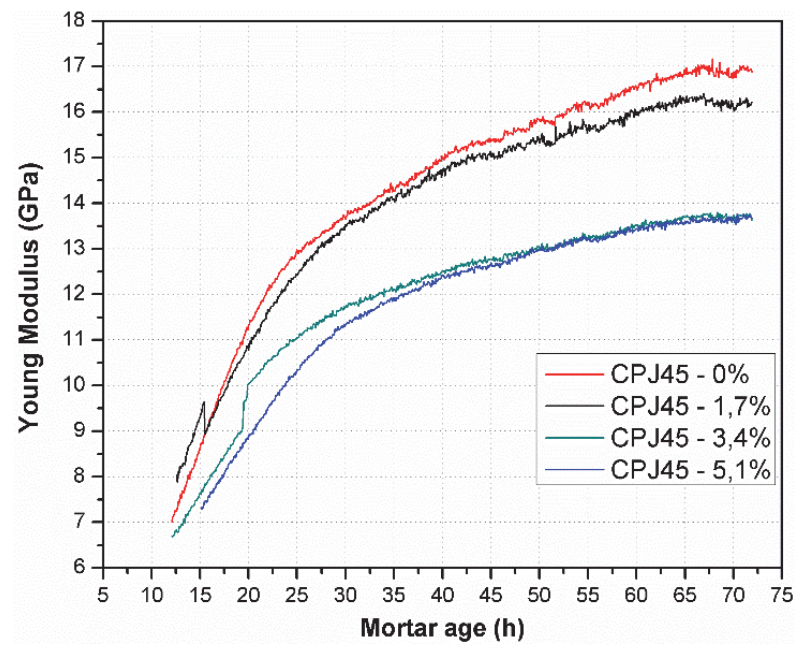

Fig. 10. Young's Modulus vs. mortar age. Mortars were prepared using Portland composite cement CPJ 45 and an alkaline accelerator dosage of $0 \%, 1.7 \%, 3.4 \%$ and $5.1 \%$ of the cement weight used

During the hardening period, all the density curves show the same pattern. A decrease until the curves levelled off, indicating a steady state for the density evolution. A stepwise increase in alkaline accelerator dosage resulted in decreasing values at which the curves levelled off. By analyzing the curves, the levelling off value for the density of mortar is characterized by a higher decrease when increasing the alkaline accelerator dosage from $0 \%$ to $1.7 \%$ and from $1.7 \%$ to $3.4 \%$, compared to a lower decrease noticed when increasing the admixture's dosage from $3.4 \%$ to $5.1 \%$. Hardened cement pastes are porous solids and it has been found that density increases with decrease of porosity and vice versa. Thus, a mortar paste mixed with an alkaline accelerator dosage of 5.1\% shows a lower density values during the steady state, causing a higher quantity of pore spaces in the hardened mortar paste. By the same process, a relatively increase of porosity is caused by the decrease of density, related to an increase of alkaline accelerator dosage.

Furthermore, Young's modulus has been calculated as an ultrasound parameter, using pulse velocity and acoustic impedance. The young's modulus evolution is characterized by a stepwise increase until the 
curves levelled off. The rate of increase was greater at early times of the hardening period but become approximately linear from $25 \mathrm{~h}$ of mortar age, until reaching a levelling off value, indicating a steady state for young modulus evolution. Results show that increasing the accelerator dosage from $1.7 \%$ to $3.4 \%$ implies a higher decrease of the levelling of value. However, no significant decrease was noted when increasing the dosage from $3.1 \%$ to $5.1 \%$. Based on the young modulus curves more or less the same classification of mixes could be made as based on the attenuation coefficient and density.

The Young's modulus indicates the resistivity of a material against deformation caused by loading. Therefore, the results confirm that the stepwise increase in alkaline accelerator dosage caused a progressive decrease in young's modulus, indicating a continuous decrease of resistivity of the hardened mortar paste. De Belie et al. (2005) found that only the alkaline accelerator caused a significant reduction in the compressive strength in comparison with the reference mortar without accelerator, and this for all the dosages tested. This is expected since there is an exponential decrease in the strength of the cementitious material with increasing porosity (Powers, 1947; Roy\& Gouda, 1975; Popovics 1969).

In addition, the ultrasound color histograms below (Figs. 11, 12, 13 and 14) represent the superposition of all the backscattered signals within $72 \mathrm{~h}$ of mortar age, for a recording interval of $5 \mathrm{~min}$ between two successive recorded signals. Those histograms show the evolution of the principal echoes contained in backscattered signals, during the setting and hardening period of mortar samples, containing the different accelerator dosages.

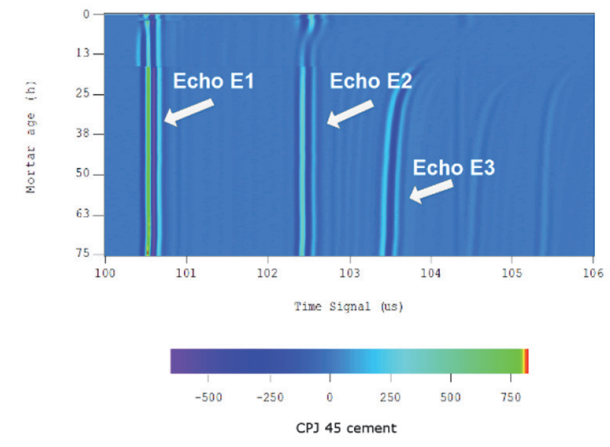

Fig. 11. Histogram of ultrasound backscattered signals data, during $72 \mathrm{~h}$ of mortar age, for reference mortar without accelerator

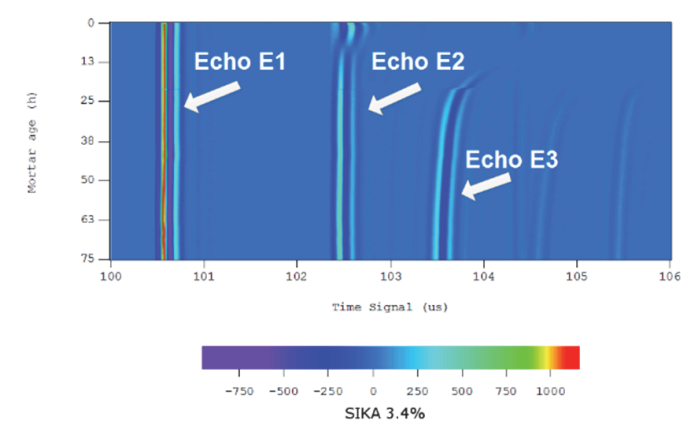

Fig. 13. Histogram of ultrasound backscattered signals data, during $72 \mathrm{~h}$ of mortar age, for mortar containing an accelerator dosage of $3.4 \%$

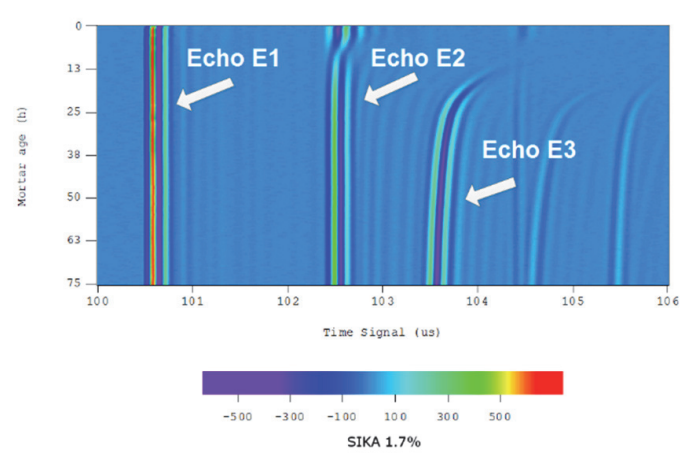

Fig. 12. Histogram of ultrasound backscattered signals data, during $72 \mathrm{~h}$ of mortar age, for mortar containing an accelerator dosage of $1.7 \%$

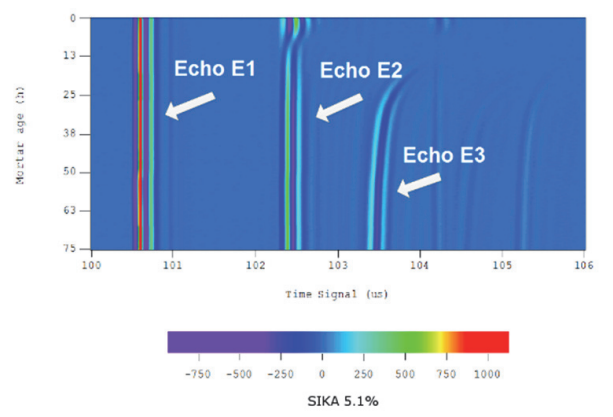

Fig. 14. Histogram of ultrasound backscattered signals data, during $72 \mathrm{~h}$ of mortar age, for mortar containing an accelerator dosage of $5.1 \%$

On all these ultrasound histograms, the first thing to notice is that the echo E3 does not begin to occur until the start of the hardening period. Thus, a stepwise increase in alkaline accelerator dosage resulted in decreasing value for the amplitude of echo E3 during the hardening period, which leads to infer that the stepwise increase in alkaline accelerator dosage is among the main causes of attenuation of the ultrasound P-wave propagating in the accelerated mortar samples. Also, on these histograms, the 
amplitude of the echo E2 decreases sharply from the beginning of the setting period, until reaching a minimum that indicates the end of setting. Then, this amplitude increases sharply from the beginning of the hardening period after which a steady state is reached. The setting period corresponds to the time range from the start of the stepwise decrease of the amplitude of echo E2, followed by reaching a minimum, until the start of the gradually increase of this amplitude, indicating the end of setting and the beginning of the hardening period. As reported in the histograms, this time interval varied as a function of alkaline accelerator dosage. In fact, a higher dosage corresponds to a shorter setting time interval.

By analyzing the evolution of the attenuation parameter and the different echoes in the histograms, in the time (mortar age) domain, we noticed just a low decrease in the setting period when rising accelerator dosage from $3.4 \%$ to $5.1 \%$. However, it is very clear that the ultrasound attenuation is sensitive to this rise from $3.4 \%$ to $5.1 \%$. Besides, one of the main causes of attenuation in cementitious materials is the expansion of its porous volume, which causes its strength to decrease. The density and young's modulus curves show that an increase in alkaline accelerator dosage from $1.7 \%$ to $3.1 \%$ causes a higher decrease during the hardening period of the two evaluated physicochemical and mechanical parameters. As a recommendation, the use of alkaline accelerator in the field should be by a dosage not exceeding $1.7 \%$ of the cement weight used in the mixture, in order to avoid a significant decrease in the strength and resistivity of the hardened cementitious material.

\section{Conclusion}

Ultrasound frequency dependent attenuation coefficient as a fundamental acoustic parameter of a material can be used to characterize mortar's behaviour at early ages and therefore help predict the cementitious material's performance on the macro scale. As a result of this study, the following of attenuation coefficient evolution of P-waves propagating in the evaluated mortar pastes, as a function of mortar age, allowed us to identify the four major stages of Portland composite cement hydration. The first stage is the rapid heat generation period, followed by the dormant period, then the setting period and after that comes the hardening period. The time when the attenuation coefficient reaches its maximum indicates the end of the setting period and the beginning of the hardening stage. Thus, the stepwise increase in alkaline accelerator dosage resulted in a more precipitated end time of setting. Also, the increase in alkaline accelerator dosage indicates a more attenuative cement-mortar medium during the hardening period. This could be due to an increase in the porosity ratio of the hardened mortar pastes. In addition, the previous results were validated by following the evolution of the different generated echoes, represented on ultrasound color histograms in time domain. Results showed also that the combined use of ultrasound P-wave measured density and young modulus parameters results in a comprehensive ultrasound method that gives a more effective results of the porosity ratio and resistivity of hardened mortar pastes.

\section{References}

ASTM standard test method for time of setting of Portland cement pastes containing accelerating admixtures for shotcrete by the use of Gillmore needles (C 1102-88). Annual book of ASTM standards, V. 02-02, 2000, Philadelphia, $568-569$

Bita, H., Faiz, B., Moudden, A., Lotfi, H., Ouacha, E. H., \& Banouni, H. (2017). Ultrasound monitoring of a mortar hydration using Argand Diagram: The effect of sand grain size and temperature. Construction and Building Materials, 155, 1003-1012.

De Belie, N., Grosse, C. U., Kurz, J., \& Reinhardt, H. W. (2005). Ultrasound monitoring of the influence of different accelerating admixtures and cement types for shotcrete on setting and hardening behaviour. Cement and Concrete Research, 35(11), 2087-2094.

Grosse, C. U., \& Reinhardt, H. W. (2000). Ultrasound technique for quality control of cementitious materials. In Proceedings of the 15th Conference on Nondestructive Testing (Vol. 15). 
Khatib, N., Ouacha H., Faiz, B., Ezzaidi M. \& Banouni, H. (2018). Ultrasound study of setting and hardening behaviour of mortar using Portland composite cements CPJ45 and CPJ35 and different dosages of an Alkalifree setting accelerator for Shotcrete. Journal of Engineering and Applied Sciences, 13, 7150-7155.

Lofti, H., Faiz, B., Moudden, A., Menou, A., Izbaim, I., Maze, G., \& Decultot, D. (2009). Ultrasonic Characterization and Hardening of Mortar Using the Reflection Technique. High Temperature Materials and Processes, 28(4), 263-270.

Lotfi, H, Moudden, A. \& Faiz, B. (2013). Processing of reflection coefficient of signals backscattered by mortar using an ultrasonic technique. American Journal of Signal Processing, 3(2), 17-24

Moroccan Industrial Standards Service (SNIMA), Binders Hydraulic-Cement-Composition, specifications and conformity criteria REV, NM 10.1.004, 24p, 2003.

T.A. Melbye, Sprayed Concrete for Rock Support, 5th ed., MBT International Underground Construction Group, Zurich, Switzerland, 1996 (188 pp.).

Pinkerton, J. M. M. (1947). A pulse method for the measurement of ultrasonic absorption in liquids: results for water. Nature, 160(4056), 128.

Popovics, S. (1969). Effect of Porosity on the Strength of Concrete. Journal of materials.

Powers, T. C. (1947). Physical properties of hardened Portland cement paste, Part 6. Relation of physical characteristics the paste to compressive strength. ACI Journal, 43(9), 845-880.

Reinhardt, H. W., \& Grosse, C. U. (2004). Continuous monitoring of setting and hardening of mortar and concrete. Construction and Building Materials, 18(3), 145-154.

Reinhardt, H. W., Grosse, C. U., \& Herb, A. T. (2000). Ultrasonic monitoring of setting and hardening of cement mortar-A new device. Materials and Structures, 33(9), 581-583.

Reinhardt, H. W., Grosse, C. W., Herb, A., Weiler, B., \& Schmidt, G. (2003). U.S. Patent No. 6,655,213. Washington, DC: U.S. Patent and Trademark Office.

Robeyst, N., Gruyaert, E., Grosse, C. U., \& De Belie, N. (2008). Monitoring the setting of concrete containing blast-furnace slag by measuring the ultrasonic p-wave velocity. Cement and Concrete research, 38(10), 11691176.

Roy, D. M., \& Gouda, G. R. (1975). Optimization of strength in cement pastes. Cement and Concrete Research, $5(2), 153-162$.

Schmerr, L. W. (2016). Fundamentals of ultrasonic nondestructive evaluation. New York: Springer.

Trtnik, G., Turk, G., Kavčič, F., \& Bosiljkov, V. B. (2008). Possibilities of using the ultrasonic wave transmission method to estimate initial setting time of cement paste. Cement and Concrete Research, 38(11), 1336-1342.

Zhu, J., Kee, S. H., Han, D., \& Tsai, Y. T. (2011). Effects of air voids on ultrasonic wave propagation in early age cement pastes. Cement and Concrete Research, 41(8), 872-881.

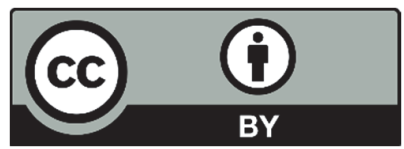

(C) 2019 by the authors; licensee Growing Science, Canada. This is an open access article distributed under the terms and conditions of the Creative Commons Attribution (CC-BY) license (http://creativecommons.org/licenses/by/4.0/). 